\title{
Auditory Neural Responses to Click Stimuli of Different Rates in the Brainstem of Very Preterm Babies at Term
}

\author{
ZE D. JIANG, DOROTHEA M. BROSI, AND ANDREW R. WILKINSON \\ Neonatal Unit, Department of Paediatrics, John Radcliffe Hospital, University of Oxford, Headington, \\ Oxford OX3 9DU, United Kingdom
}

\begin{abstract}
ABSTRA
Auditory neural responses to acoustic stimuli of different rates
were studied by analyzing changes in brainstem auditory evoked
responses (BAER) with increasing repetition rate of clicks, or
rate-dependent changes, in 62 very preterm babies (gestation
$24-32$ wk). None had perinatal asphyxia or major complications
at the time of testing (37-42 wk postconceptional age) to exclude
their possible effects on the recorded BAER. As the rate of clicks
was increased from $21 / \mathrm{s}$ to $51 / \mathrm{s}$ and $91 / \mathrm{s}$, I-V interpeak interval
in these babies increased similarly to that in normal term neo-
nates. The rate-dependent change decreased significantly in I-III
interval, but increased significantly in III-V intervals and III-V/
I-III interval ratio (all $p<0.01$ ). At all three rates of clicks, the
I-V interval, the most commonly used BAER variable, tended to
increase slightly but did not differ significantly from the term
neonates. The I-III interval decreased significantly at higher
click rates (ANOVA $p<0.05$ at $51 / \mathrm{s}$ and $<0.001$ at $91 / \mathrm{s}$ ),
\end{abstract}
Babies who are born very preterm account for a large proportion of perinatal mortality and are at high risk of neurodevelopmental deficits (1-5). Many of the survivors have long-term neurologic disorders or behavior problems, e.g. minimal brain damage and learning disabilities, and visual and hearing deficits. Those who have sensorineural hearing loss develop more neurologic complications than control infants (6). However, there are still controversies regarding early neural development in these babies. Some investigators reported a delay in development (7-9), whereas others found no apparent abnormality $(10,11)$. Better understanding of the early development and neurophysiology underlying later disability is important for improving the management of these babies.

BAERs represent the electrophysiologic activity of a large number of auditory neurons in the brainstem after transient

Received December 4, 2000; accepted October 2, 2001.

Correspondence and reprint requests: Ze Dong Jiang, M.D., Neonatal Unit, Department of Paediatrics, John Radcliffe Hospital, University of Oxford, Headington, Oxford OX3 9DU, United Kingdom; e-mail: zedong.jiang@paediatrics.ox.ac.uk

Supported by the Children Nationwide Medical Research Fund, Babes in Arms, Defeating Deafness, and the Wellcome Trust of United Kingdom. whereas the III-V interval and III-V/I-III interval ratio increased significantly at all $21 / \mathrm{s}, 51 / \mathrm{s}$, and particularly $91 / \mathrm{s}(p<0.01-$ 0.001 ). No abnormalities were found in wave $\mathrm{V}$ amplitude at any rates. These results suggest that very preterm babies have an advanced peripheral development of the brainstem auditory pathway but a retarded central development or central impairment. A nearly normal $\mathrm{I}-\mathrm{V}$ interval does not exclude a possible abnormality in its subcomponents (I-III and III-V intervals). In babies who have a normal BAER at a conventionally used low rate of clicks, we cannot exclude an abnormal BAER at higher rates.

(Pediatr Res 51: 454-459, 2002)
Abbreviations
BAER, brainstem auditory evoked response dB $\mathrm{nHL}$, decibel normal hearing level

acoustic stimulation. The responses have been widely used to study auditory function and its development in babies (12-18). Although most of the previous BAER studies in preterm babies reported no significant abnormalities $(12,18)$, others reported an increase in BAER wave latencies and intervals, suggesting retarded development (16). To date, none of the previous reports were concentrated on the study of BAER central components in very preterm babies.

The neonatal BAER is significantly affected by the repetition rate of acoustic stimuli to elicit it $(13,15,19)$. Routinely used relatively low rates of stimuli (usually $10-21 / \mathrm{s}$ ) can elicit a clear, easily identifiable BAER waveform. However, a stimulus condition that is optimal for eliciting the most easily identifiable BAER waveform may not be optimal for demonstrating pathology. Increasing the rate of stimuli used while recording the BAER provides a more stressful stimulation, or a stronger physiologic challenge, to the auditory neurons than low-rate stimulation. It has been suggested by some authors as a stress test or challenge test to improve the detection of auditory impairment and other neuropathology of the brain, although there are some debates (15, 20-26). Furthermore, 
changes in the BAER with increasing the rate of stimuli, or rate-dependent changes, primarily reflect neural processes concerning the efficacy of synaptic transmission and metabolic status of auditory neurones after a temporal challenge $(13,19$, 20). Therefore, the method of increasing stimulus rate has also been used to study dynamic properties of the auditory system and their development.

Previous studies of the BAER in preterm babies often used only one low rate of click stimuli (10-21/s). It is not clear whether there is any abnormality in the BAER at high rates of stimuli in very preterm babies. To understand auditory neural responses to acoustic stimuli of different rates in very preterm babies we have studied the BAER elicited with different rates of clicks and the rate-dependent changes in babies who were born younger than 33 wk of gestation. None had perinatal asphyxia or any major complications at the time of testing (term equivalent date) to exclude any possible effects of these factors on the recorded BAER. Our hypothesis is that auditory responses to high-rate stimulation in very preterm babies is altered at term owing to the earlier exposure to sound environment ex utero and the associated perinatal conditions. A further purpose is to examine whether high-rate stimulation can improve the detection of BAER abnormalities.

\section{METHODS}

Subjects. The study group consisted of 62 very preterm babies, 34 boys and 28 girls, who were born at $24-32 \mathrm{wk}$ (mean, $28.0 \pm 2.2 \mathrm{wk}$ ) of gestation, with a birth weight between 559 and $1999 \mathrm{~g}$ (mean, $1110 \pm 328 \mathrm{~g}$ ). Apgar scores were $8-10$ at 1 and $5 \mathrm{~min}$. Babies were excluded if they had perinatal asphyxia and major complications (e.g. chronic lung disease, infection) at the time of BAER testing, which was conducted between 37 and $42 \mathrm{wk}$ (mean, $39.6 \pm 1.8 \mathrm{wk}$ ) of postconceptional age. Those who had significant peripheral hearing loss, indicated by a significant increase in BAER threshold $(>30 \mathrm{~dB} \mathrm{nHL})$ at the time of testing, were also excluded to avoid the influence of the peripheral loss on central components of the BAER.

The term control group had 42 healthy neonates, 19 boys and 23 girls, ranging in a gestation between 37 and $41 \mathrm{wk}$ (mean, $39.4 \pm 1.3$ wk) and a birth weight between 2633 and $4559 \mathrm{~g}$ (mean, $3546 \pm 554 \mathrm{~g}$ ). All had an Apgar score 8-10 at 1 and $5 \mathrm{~min}$ and were judged to be stable and healthy in the nursery. None had any major perinatal conditions such as asphyxia, periventricular hemorrhage, intrauterine or postnatal infection, or congenital abnormality of the CNS. BAER recording was performed 1-3 d after birth. BAER thresholds were all $<20 \mathrm{~dB}$ nHL.

BAER recording procedures. The equipment and procedures for recording the BAER have been reported previously (19). Briefly, the BAER was recorded using a Bravo Portable Evoked Potential System (Nicolet Biomedical Inc., Madison, WI, U.S.A.) in a quiet room. Three gold-plated disk electrodes were placed at the middle forehead $(+)$, the ipsilateral earlobe $(-)$, and the contralateral earlobe (ground). Interelectrode impedances were maintained $<10 \mathrm{k} \Omega$. Acoustic stimuli were rarefaction clicks of $100 \mu \mathrm{s}$ in duration, delivered monaurally to the left ear through a TDH 39 earphone. Three repetition rates of clicks were presented for each subject and in the order of $21 / \mathrm{s}, 51 / \mathrm{s}$, and $91 / \mathrm{s}$ in the first run and in reverse order in the second run. Click intensities ranged between 10 and $80 \mathrm{~dB}$ $\mathrm{nHL}$, referred to average thresholds in adults (19). All subjects were tested at $60 \mathrm{~dB}$ nHL. In those with a threshold $>20 \mathrm{~dB}$ $\mathrm{nHL}$, the intensity was increased to 70 and $80 \mathrm{~dB}$ nHL to elicit clear waveforms and to obtain the data at an intensity above their thresholds that was comparable with that in the term control babies. Intensities $<60 \mathrm{~dB} \mathrm{nHL}$ were used to obtain threshold.

Brain responses to the clicks were amplified and bandpass filtered between 100 and $3000 \mathrm{~Hz}$ before inputting to the averager. An automatic artifact rejection was used to reduce the inclusion of high-amplitude muscular activity in the averaged responses. Sampling was manually discontinued whenever there were excessive muscle artifacts on the monitoring screen. Brain responses to 2048 clicks were averaged to complete each run. At least two runs were made for the recordings at $60 \mathrm{~dB} \mathrm{nHL}$.

This study was approved by the Central Oxford Research Ethics Committee. Written informed consent was obtained from the parents of each baby before study entry.

BAER measurement. Latency and amplitude of waves I, III, and $\mathrm{V}$ were measured. Interpeak intervals (I-V, I-III, and III-V), the interval ratio of III-V to I-III (or III-V/I-III interval ratio), and the amplitude ratio of waves V to I and III (or V/I and V/III amplitude ratios) were calculated and examined in detail.

Mean measurements of two replicated BAER recordings to each stimulus condition were used for data analyses. Analysis of BAER interval and amplitude variables in the term control infants was based on the data collected at the click intensity of $60 \mathrm{~dB}$ nHL. In the very preterm babies the analysis was based on the data collected at $60 \mathrm{~dB}$ nHL for thresholds $\leq 20 \mathrm{~dB} \mathrm{nHL}$ $(n=54)$ or $70 \mathrm{~dB} n H L$ for thresholds $>20-30 \mathrm{~dB}$ nHL $(n=$ 8 ), so that as in the term control babies, all data were analyzed at a hearing level $\geq 40 \mathrm{~dB}$ above the thresholds.

Statistical analysis. Correlation and regression analyses were performed between BAER measurements and the repetition rate of clicks. Mean and SD of each BAER variable at each stimulus condition were calculated. The data obtained from the very preterm babies and those from the term control babies were compared using ANOVA.

\section{RESULTS}

BAER recorded at different rates of clicks. The measurements of BAER variables at various rates of clicks were given in Tables 1 and 2. As the rate was increased all BAER wave latencies and interpeak intervals increased, and wave amplitudes decreased. The general changes in the very preterm babies were similar to those in the term control babies, but there were some differences.

Wave $\mathrm{V}$ latency in the very preterm babies increased slightly at all 21/s, 51/s, and 91/s clicks, compared with that in the term control infants (all $p<0.05$; Table 1). Wave I latency also 
tended to increase slightly. However, wave III latency did not differ from the term control babies at any of the rates.

The $\mathrm{I}-\mathrm{V}$ interval in the very preterm babies increased slightly at all rates used here, but did not differ significantly from that in the control babies (Table 1). Although the I-III interval was similar to that in the control babies at $21 / \mathrm{s}$ clicks, it decreased significantly at $51 / \mathrm{s}$ and $91 / \mathrm{s}$ clicks $(p<0.05$, $0.001)$. The III-V interval and the III-V/I-III interval ratio increased significantly at all the three rates, particularly $91 / \mathrm{s}$ (all $p<0.01-0.001$ ). Thus, the differences between the very preterm babies and the term control babies in these interval variables increased with the increase in click rate.

Compared with that in the term control babies, the amplitude of wave I in the very preterm babies decreased slightly, whereas the amplitudes of waves III and V tended to increase slightly at all 21/s, 51/s, and 91/s clicks (Table 2). As a result, the V/I amplitude ratio increase slightly, with a statistical significance at $91 / \mathrm{s}$ clicks $(p<0.05)$, and the V/III amplitude ratio was similar to that in the term control babies.

To find out whether the differences in the BAER between the very preterm babies and the term control babies have any relationship with the possible difference in their head size, we compared their head circumferences at the time of testing. The results showed that head circumference in the very preterm babies $(34.8 \pm 2.42 \mathrm{~cm}, 29.0-41.6 \mathrm{~cm})$ did not differ significantly from that in the control babies $(35.4 \pm 1.62 \mathrm{~cm}$, $32.5-8.0 \mathrm{~cm}$ ). Head circumference did not correlate with any BAER variables in the control babies and only correlated weakly with wave III and V latencies at $21 / \mathrm{s}$ clicks $(r=-0.40$ and -0.41 , both $p<0.05$ ) in the very preterm babies.

Click rate-dependent changes in the BAER. Correlation and regression analyses showed that all BAER wave latencies and interpeak intervals in the very preterm babies correlated positively and significantly with the rate of clicks. Although the general trend was similar to that in the control babies, there were differences in the rate-dependent changes, as shown by the slopes of the latency- or interval-rate functions (Table 3).

Compared with those in the control babies, the slopes of latency-rate functions for waves I and III in the very preterm babies decreased significantly ( $p<0.05,0.01)$, but the slope for wave $\mathrm{V}$ was similar to that in the control babies. The slope of interval-rate function for I-III decreased significantly $(p<$ $0.01)$. On the contrary, the slope for III-V increased significantly $(p<0.01)$. As a result, the slope for the III-V/I-III interval ratio increased significantly $(p<0.01)$.

All wave amplitudes correlated negatively and significantly with the rate of clicks (Table 4). No significant difference was found between the very preterm babies and term control babies in any of the slopes of amplitude-rate functions for waves I, III, and V. Similar to those in the term control babies, both the V/I and V/III amplitude ratios in the very preterm babies did not correlate with the rate.

\section{DISCUSSION}

Peripheral and central auditory pathways do not develop uniformly in very preterm babies. In the study of BAER in preterm babies most authors reported no significant abnormalities $(12,18)$. We have also previously compared the BAER between preterm and term babies at the same postconceptional age up to $6 \mathrm{y}$ of age and found no major differences (17). However, some others reported an increase in BAER wave latencies and intervals in preterm infants when they reached term (16). The latency for early component P0 in middle latency auditory evoked response increased, and the latencies of $\mathrm{Na}$ and $\mathrm{P} 2$ in auditory cortical evoked responses also differed significantly from those in term babies. These results suggest that myelination of the central auditory pathway is retarded in preterm babies. In the present study of very preterm babies at term, the $\mathrm{I}-\mathrm{V}$ interval tended to increase slightly but did not differ significantly from that in the term babies. It appears that there is no apparent abnormality in the auditory brainstem. This is comparable with the finding by some magnetic resonance studies that there was no significant difference in myelination stage between very preterm and term babies at term (10).

The present study found that in the very preterm babies, wave I latency increased slightly and wave I amplitude decreased slightly. On the other hand, the rate-dependent change in wave I latency decreased. These results suggest that periph-

Table 1. BAER latencies and interpeak intervals at different rates of clicks ( $\geq 40 \mathrm{~dB}$ above BAER threshold)

\begin{tabular}{lllll}
\hline BAER variables & \multicolumn{1}{c}{ Subjects } & \multicolumn{1}{c}{$21 / \mathrm{s}$} & \multicolumn{1}{c}{$51 / \mathrm{s}$} & $91 / \mathrm{s}$ \\
\hline I (ms) & Very preterm & $2.42 \pm 0.16^{*}$ & $2.62 \pm 0.19$ & $2.77 \pm 0.20$ \\
& Normal term & $2.33 \pm 0.21$ & $2.53 \pm 0.22$ & $2.70 \pm 0.25$ \\
III (ms) & Very preterm & $5.10 \pm 0.26$ & $5.39 \pm 0.30$ & $5.59 \pm 0.32$ \\
V (ms) & Normal term & $5.08 \pm 0.24$ & $5.37 \pm 0.26$ & $5.66 \pm 0.26$ \\
& Very preterm & $7.40 \pm 0.33^{*}$ & $7.83 \pm 0.37^{*}$ & $8.29 \pm 0.39^{*}$ \\
I-III (ms) & Normal term & $7.22 \pm 0.23$ & $7.69 \pm 0.29$ & $8.14 \pm 0.30$ \\
& Very preterm & $2.67 \pm 0.24$ & $2.75 \pm 0.26^{*}$ & $2.78 \pm 0.26^{* * *}$ \\
III-V (ms) & Normal term & $2.74 \pm 0.17$ & $2.83 \pm 0.19$ & $2.97 \pm 0.17$ \\
& Very preterm & $2.29 \pm 0.19^{* *}$ & $2.45 \pm 0.21^{* *}$ & $2.72 \pm 0.23^{* * *}$ \\
I-V (ms) & Normal term & $2.16 \pm 0.11$ & $2.33 \pm 0.12$ & $2.49 \pm 0.14$ \\
III-V/I-III ratio & Very preterm & $4.97 \pm 0.27$ & $5.21 \pm 0.31$ & $5.51 \pm 0.33$ \\
& Normal term & $4.90 \pm 0.17$ & $5.16 \pm 0.22$ & $5.46 \pm 0.19$ \\
& Very preterm & $0.87 \pm 0.13^{* *}$ & $0.92 \pm 0.13^{* *}$ & $0.99 \pm 0.13^{* * *}$ \\
\hline
\end{tabular}

Values are mean $\pm \mathrm{SD}$.

$* p<0.05 ; * *<0.01 ; * * *<0.001$ by ANOVA for comparison between the very preterm babies and the normal term control babies. 
Table 2. BAER amplitude variables at different rates of clicks ( $\geq 40 \mathrm{~dB}$ above BAER threshold)

\begin{tabular}{|c|c|c|c|c|}
\hline $\begin{array}{c}\text { BAER } \\
\text { variables }\end{array}$ & Subjects & $21 / \mathrm{s}$ & $51 / \mathrm{s}$ & $91 / \mathrm{s}$ \\
\hline \multirow[t]{2}{*}{$\mathrm{I}(\mu \mathrm{V})$} & Very preterm & $0.175 \pm 0.064 *$ & $0.152 \pm 0.051 *$ & $0.114 \pm 0.047 *$ \\
\hline & Normal term & $0.204 \pm 0.048$ & $0.171 \pm 0.047$ & $0.137 \pm 0.042$ \\
\hline \multirow[t]{2}{*}{ III $(\mu \mathrm{V})$} & Very preterm & $0.246 \pm 0.089$ & $0.223 \pm 0.070 *$ & $0.181 \pm 0.060$ \\
\hline & Normal term & $0.218 \pm 0.066$ & $0.187 \pm 0.049$ & $0.170 \pm 0.056$ \\
\hline \multirow[t]{2}{*}{$\mathrm{V}(\mu \mathrm{V})$} & Very preterm & $0.203 \pm 0.067$ & $0.187 \pm 0.048$ & $0.163 \pm 0.057$ \\
\hline & Normal term & $0.198 \pm 0.051$ & $0.178 \pm 0.048$ & $0.156 \pm 0.037$ \\
\hline \multirow[t]{2}{*}{$\mathrm{V} / \mathrm{I}$ ratio } & Very preterm & $1.450 \pm 0.123$ & $1.272 \pm 0.485$ & $1.739 \pm 1.040 *$ \\
\hline & Normal term & $1.038 \pm 0.340$ & $1.146 \pm 0.440$ & $1.203 \pm 0.394$ \\
\hline \multirow[t]{2}{*}{$\mathrm{V} / \mathrm{III}$ ratio } & Very preterm & $0.934 \pm 0.353$ & $0.896 \pm 0.294$ & $1.047 \pm 0.382$ \\
\hline & Normal term & $1.016 \pm 0.370$ & $0.988 \pm 0.353$ & $1.004 \pm 0.397$ \\
\hline
\end{tabular}

Values are mean $\pm \mathrm{SD}$.

$* p<0.05$; by ANOVA for comparison between the very preterm babies and the normal term control babies.

Table 3. Regression analysis of the relationship between BAER latency and interval variables and click rate

\begin{tabular}{|c|c|c|c|c|}
\hline BAER variables & Subjects & Intercept & Slope (/decade) & $\begin{array}{c}\text { Slope }=0 \\
p \text { value of } t \text { test* }\end{array}$ \\
\hline \multirow[t]{2}{*}{ I (ms) } & Very preterm & $2.370 \pm 0.034$ & $0.045 \pm 0.006$ & $<0.001$ \\
\hline & Normal term & $2.245 \pm 0.071$ & $0.051 \pm 0.012$ & $<0.001$ \\
\hline \multirow[t]{2}{*}{ III (ms) } & Very preterm & $5.053 \pm 0.053$ & $0.060 \pm 0.009$ & $<0.001$ \\
\hline & Normal term & $4.975 \pm 0.071$ & $0.073 \pm 0.012$ & $<0.001$ \\
\hline \multirow[t]{2}{*}{ V (ms) } & Very preterm & $7.237 \pm 0.067$ & $0.129 \pm 0.011$ & $<0.001$ \\
\hline & Normal term & $6.981 \pm 0.078$ & $0.131 \pm 0.013$ & $<0.001$ \\
\hline \multirow[t]{2}{*}{ III-V (ms) } & Very preterm & $2.181 \pm 0.037$ & $0.058 \pm 0.006$ & $<0.001$ \\
\hline & Normal term & $2.056 \pm 0.033$ & $0.046 \pm 0.005$ & $<0.001$ \\
\hline \multirow[t]{2}{*}{$\mathrm{I}-\mathrm{V}(\mathrm{ms})$} & Very preterm & $4.847 \pm 0.050$ & $0.073 \pm 0.008$ & $<0.001$ \\
\hline & Normal term & $4.762 \pm 0.054$ & $0.075 \pm 0.009$ & $<0.001$ \\
\hline \multirow[t]{2}{*}{ III-V/I-III ratio } & Very preterm & $0.843 \pm 0.021$ & $0.016 \pm 0.004$ & $<0.001$ \\
\hline & Normal term & $0.776 \pm 0.020$ & $0.008 \pm 0.003$ & $<0.050$ \\
\hline
\end{tabular}

Values are mean \pm SEM.

* Two-tailed $t$ test.

Table 4. Regression analysis of the relationship between BAER amplitude variables and click rate

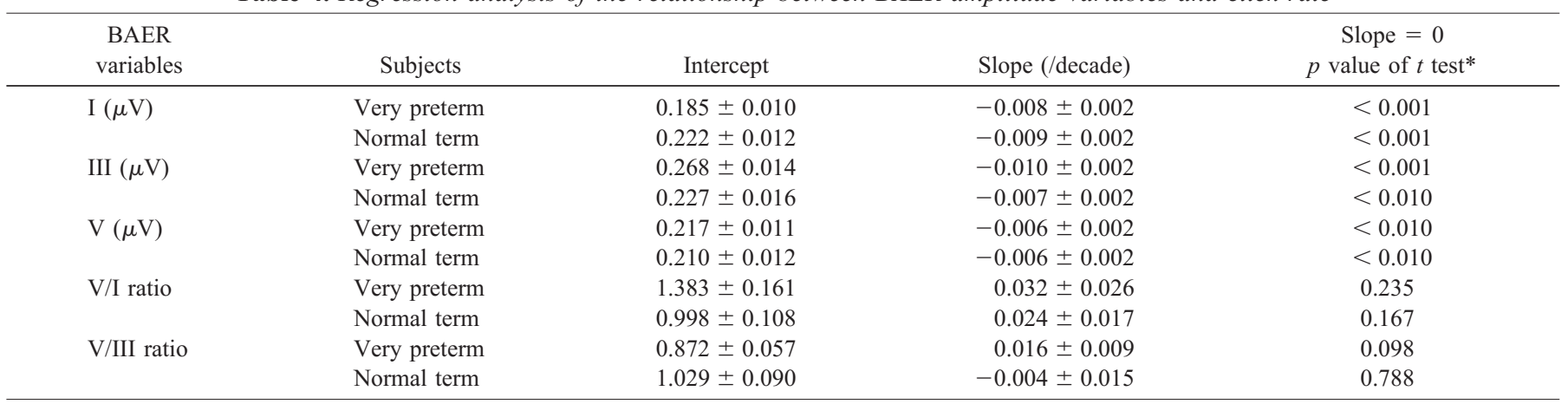

Values are mean \pm SEM.

* Two-tailed $t$ test.

eral auditory function is mildly impaired in very preterm babies but its development is accelerated, or advanced. This outcome may be attributed to by very preterm birth, which exposes the babies to sound environment ex utero earlier than term babies, and the associated perinatal conditions, which may impair peripheral auditory function.

Although the rate-dependent change in the $\mathrm{I}-\mathrm{V}$ interval in the very preterm babies was similar to that in the term babies, the rate-dependent changes in the subcomponents (I-III and III-V intervals) of the I-V interval in the very preterm babies differed from those in the term babies: the rate-dependent change was decreased in the I-III interval but increased in the III-V interval. The differences suggest that the I-III interval in the very preterm babies changes little with varying click rate but the III-V interval changes more significantly than in the term babies. The decreased rate-dependent change in the I-III interval was produced by a more significant decrease in the rate-dependent change in wave III latency than in wave I latency. The increased change in the III-V interval was produced by a decrease in the rate-dependent change in wave III latency and a net increase in the change in wave V latency (after cancelling the cumulative effect of the decreased change 
in wave III latency). Thus, the opposite rate-dependent changes in the two intervals were both partly related to the decreased rate-dependent change in wave III latency.

These BAER abnormalities are unlikely to be related to the head size in the very preterm babies because none of the intervals correlate with head circumference in these babies and there was no significant difference between the very preterm and term babies in head circumference.

It is known that waves I and II originate exclusively from the eighth nerve, wave III is mainly generated by the neurons in the cochlear nucleus, and wave $\mathrm{V}$ is most likely originated by the lateral lemniscus as it enters the inferior colliculus, although the slow negative potential after the peak of wave $\mathrm{V}$ is likely a dendritic potential of the inferior colliculus (23). The I-III interval reflects functional status of the more peripheral portion of the brainstem auditory pathway whereas the III-V interval reflects functional status of the more central portion of the pathway. Thus, the difference in the rate-dependent changes in various BAER variables of the very preterm babies imply that the peripheral and central portions of this pathway do not develop uniformly. The decreased rate-dependent changes in the I-III interval and wave I and III latencies, which are more adultlike (19), suggest an advanced development in the more peripheral portion of the brainstem auditory pathway, probably related to the earlier exposure to sound environment ex utero, which may accelerate peripheral auditory development (17). On the other hand, the increased rate-dependent change in the III-V interval indicates a retarded development or impairment in the more central portion of the pathway, probably mainly related to the associated perinatal conditions that may impair central auditory function and delay its development. However, considering that the $\mathrm{I}-\mathrm{V}$ interval changes little at various click rates, we cannot exclude a possibility that a possible change in the morphology of the waveform at the latency of wave III at high-rate stimulation could contribute to the decreased rate-dependent change in wave III latency, which, in turn, results in a decreased rate-dependent change in the I-III interval and an increased change in the III-V interval.

Very preterm birth and perinatal asphyxia have different effects on auditory function. In term asphyxiated babies, we have found that the measurements of I-III interval at 21/s, $51 / \mathrm{s}$, and $91 / \mathrm{s}$ were all similar to the normal control babies, although the I-V and III-V intervals consistently increased (21). At high-rate stimulation, the amplitude of wave $\mathrm{V}$ reduced significantly, the V/I amplitude ratio decreased, and the ratedependent change in the amplitude of wave $\mathrm{V}$ increased significantly. These results indicate that the neural generators of wave $\mathrm{V}$ after hypoxic-ischemic insult are particularly vulnerable to a physiologic challenge $(20,21)$. By contrast, the present study showed that in very preterm babies the I-III interval decreased significantly at high-rate stimulation but the amplitude of wave $\mathrm{V}$ did not differ from the normal control babies at all three click rates (the V/I amplitude ratio even increased at 91/s clicks), suggesting that the generators of wave $\mathrm{V}$ in these babies are not significantly affected. It appears that very preterm birth and perinatal asphyxia have different effects on the auditory pathway. Very preterm birth has a major effect on neural conduction of the pathway, probably reflecting al- tered myelination owing to the earlier exposure to sound environment ex utero (resulting in advanced peripheral development) and the associated perinatal conditions (resulting in retarded central development or central impairment). By contrast, asphyxia has a major effect on functional status of neural generators of wave $\mathrm{V}$, mainly related to synaptic inefficiency or asynchronous firing of neurons owing to hypoxic-ischemic neuronal damage, which becomes apparent after a physiologic challenge (high-rate stimulation).

Value of I-III and III-V intervals in the detection of auditory abnormality. The $\mathrm{I}-\mathrm{V}$ interval, the so-called brainstem conduction time and the most commonly used BAER variable reflecting neural function of the auditory brainstem, is the sum of the I-III and III-V intervals. On the basis of the finding of a normal I-V interval in the very preterm babies at all the rates of clicks used here, it appears that the general responses of auditory neurons to acoustic stimuli of different rates are normal in these babies. Nevertheless, analyses of the subcomponents of this interval revealed that there were abnormalities in the I-III and III-V intervals. Therefore, an apparently or nearly normal $\mathrm{I}-\mathrm{V}$ interval does not exclude a possible abnormality in its subcomponents (I-III and III-V intervals). These findings also indicate that the I-III and III-V intervals (and their ratio III-V/I-III) are useful BAER variables that can reveal some abnormalities that cannot be shown by the $\mathrm{I}-\mathrm{V}$ interval.

Why did the very preterm babies show abnormalities in both I-III and III-V intervals at high-rate stimulation but no apparent abnormalities in the I-V interval? This can be explained by the mutual effects of the opposite changes in the I-III and III-V intervals. The earlier subcomponent (I-III interval) of the I-V interval decreased significantly at high click rates, but the later subcomponent (III-V interval) increased significantly, particularly at higher rates. As a result, the I-V interval showed no significant change at all rates used here.

Method of increasing stimulus rate while recording the BAER can improve the detection of auditory abnormality. There are still debates regarding whether this method is valid to improve the detection of auditory abnormalities $(15,20-26)$. We have also previously studied the BAER, obtained with various repetition rates, and have found that auditory neuropathology in some cases may not be significant enough to be detected by the BAER with low-rate stimulation, but can become detectable with high-rate stimulation $(15,20,21,25$, 26). The present study showed that the differences between the very preterm babies and the term control babies in the I-III and III-V intervals increased with the increase in click rate: the I-III interval became abnormal at high rates, whereas the abnormalities in the III-V interval and III-V/I-III interval ratio became more significant at the higher rates.

Furthermore, the rate-dependent change provides a further index for the assessment of auditory neural function. In this study, the finding that very preterm babies have an advanced peripheral development of the brainstem auditory pathway but a retarded central development or central impairment was mainly derived from the results of the rate-dependent changes in different BAER variables. 
These results suggest that increasing the rate of clicks while recording the BAER is a valuable method to improve the detection of auditory neuropathology. An apparently normal or nearly normal BAER in response to routinely used low-rate stimulation may be abnormal when a more stressful high-rate stimulation is used. However, there is a disadvantage of increasing stimulus rate, in that BAER waveform morphology tends to deteriorate at rates higher than $51 / \mathrm{s}$, which sometimes makes it difficult to measure BAER variables accurately and reliably.

The present study indicates that in babies who have a normal BAER recorded with conventional low-rate stimulation, we cannot rule out possible auditory abnormalities, which may be revealed only with high-rate stimulation. Similar to our findings in asphyxiated babies (21), a significant increase in the rate, e.g. $91 / \mathrm{s}$, is more effective to increase the detection of abnormalities than a moderate increase, e.g. 51/s. Thus, to improve the detection of neuropathology by increasing repetition rate of stimuli, the rate used should be high enough to effectively stress the large number of neurons along the brainstem auditory pathway.

Acknowledgments. The authors thank the nurses and doctors at the Neonatal Unit of the John Radcliffe Hospital for their assistance in recruiting babies.

\section{REFERENCES}

1. Wood NS, Marlow N, Costeloe K, Gibson AT, Wilkinson AR 2000 Neurologic and developmental disability after extremely preterm birth. EPICure Study Group. N Engl J Med 343:378-384

2. Stewart AL, Rifkin L, Amess PN, Kirkbride V, Townsend JP, Miller DH, Lewis SW, Kingsley DP, Moseley IF, Foster O, Murray RM 1999 Brain structure and neurocognitive and behavioural function in adolescents who were born very preterm. Lancet 353:1653-1657

3. Veen S, Sassen ML, Schreuder AM, Ens Dokkum MH, Verloove Vanhorick SP, Brand R, Grote JJ, Ruys JH 1993 Hearing loss in very preterm and very low birthweight infants at the age of 5 years in a nationwide cohort. Int J Pediatr Otorhinolaryngol 26:11-28

4. Cooke RWI 1996 Factors affecting survival and development in extremely tiny babies. Semin Neonatal 1:267-276

5. Hagan R, Benninger H, Chiffings D, Evans S, French N 1996 Very preterm birth-a regional study. Part 2: the very preterm infants. Br J Obstet Gynaecol 103:239-245

6. Marlow ES, Hunt LP, Marlow N 2000 Sensorineural hearing loss and prematurity. Arch Dis Child 82:F141-F144
7. Takashima S, Laurence EB, Chan F 1982 Retardation of neuronal maturation in premature infants compared with term infants of the same post CA. Pediatrics 69:33-39

8. Dietrich RB, Bradley WG, Zaragoza IV EJ, Taira RK, Wilson GH, Kabgarloo H 1986 MR evaluation of early myelination patterns in normal and developmentally delayed infants. Am J Neurosci Res 9:69-76

9. McArdle CB, Richardson CJ, Nicholas DA, Mirfakhraee M, Hayden CK, Amparo EG 1987 Developmental features of the neonatal brain: MR imaging. Part I: gray-white matter differentiation and myelination. Radiology 162:395-403

10. Van de Bor M, Guit GL, Schreuder AM, van Bel F, Wondergem J, den Ouden L, Vielvoye GJ 1990 Does very preterm birth impair myelination of the central nervous system? Neuropediatrics 21:37-39

11. Konishi Y, Hayakawa K, Kuriyama M, Fujii Y, Sudo M, Konishi K, Ishii Y 1993 Developmental features of the brain in preterm and full term infants in MR imaging. Early Hum Dev 34:155-162

12. Fawer C-L, Dubowitz LMS 1982 Auditory brain stem response in neurologically normal preterm and fullterm newborn infants. Neuropediatrics 13:200-206

13. Ken-Dror A, Pratt H, Zeltzer M, Sujov P, Katzir J, Benderley A 1987 Auditory brainstem evoked potentials to clicks at different presentation rates: estimating maturation of pre-term and full-term neonates. Electroencephalog Clin Neurophysiol 68:209-218

14. Eggermont JJ, Salamy A 1988 Maturational time course for the ABR in preterm and full term infants. Hear Res 33:35-48

15. Henderson-Smart DJ, Pettigrew AG, Edwards DA, Jiang ZD 1991 Brain stem auditory evoked responses: physiological and clinical issues. In: Hanson MA (ed) The Fetal and Neonatal Brain Stem: Developmental and Clinical Issues. Cambridge University Press, Cambridge, pp 211-229

16. Pasman JW, Rotteveel JJ, de Graaf R, Stegeman DF, Visco YM 1992 The effect of preterm birth on brainstem, middle latency and cortical auditory evoked responses (BMC AERs). Early Hum Dev 31:113-129

17. Jiang ZD 1995 Maturation of the auditory brainstem in low risk preterm infants: a comparison with age-matched full term infants up to 6 years. Early Hum Dev $42: 49-65$

18. Rotteveel JJ, de Graaf R, Colon EJ, Stegeman DF, Visco YM 1986 The maturation of the central auditory conduction in preterm infants until three months post term. II: the auditory brainstem responses (ABRs). Hear Res 26:21-35

19. Jiang ZD, Brosi DM, Wilkinson AR 1998 Immaturity in electrophysiological response of the neonatal auditory brainstem to high repetition rates of click stimulation. Early Hum Dev 52:133-143

20. Jiang ZD, Brosi DM, Shao XM, Wilkinson AR 2000 Maximum length sequence brainstem auditory evoked response in infants after perinatal hypoxia-ischaemia. Pediatr Res 48:639-645

21. Jiang ZD, Brosi DM, Liu XY, Wilkinson AR 2001 Comparison of brainstem auditory evoked responses recorded at different presentation rates of clicks in term neonates after asphyxia. Acta Paediatr 90:1416-1420

22. Gerling IJ, Finitzo-Hieber T 1983 Auditory brainstem response with high stimulus rates in normal and patient populations. Ann Otol Rhinol Laryngol 92:119-123

23. Chiappa KH 1990 Brainstem auditory evoked potentials: methodology. In: Chiappa KH (ed) Evoked Potentials in Clinical Medicine. Raven Press, New York, pp $173-221$

24. Freeman S, Sohmer H, Silver S 1991 The effect of stimulus repetition rate on the diagnostic efficacy of the auditory nerve-brain-stem evoked response. Electroencephalog Clin Neurophysiol 78:284-290

25. Jiang ZD 1999 Outcome of brainstem auditory electrophysiology in children who survived purulent meningitis. Ann Otol Rhinol Laryngol 108:429-434

26. Jiang ZD, Brosi DM, Wilkinson AR 2001 Neural responses to different rates of click stimuli in the brainstem of preterm babies less than 33 weeks of gestation. Pediatr Res 49:372A(abstr) 